\title{
Tongue Alveolar Soft Part Sarcoma'
}

National Cancer Institute

\section{Source}

National Cancer Institute. Tongue Alveolar Soft Part Sarcoma'. NCI Thesaurus. Code C156273.

An alveolar soft part sarcoma involving the tongue. It usually occurs in children. 